Katrin Stump

\title{
Kooperation unter dem Dach einer starken Marke. Die Zusammenarbeit der Bibliotheken der TU9
}

Die Digitalisierung in Forschung, Lehre und Gesellschaft stellt wissenschaftliche Bibliotheken und weitere Infrastruktureinrichtungen vor enorme Herausforderungen, die sie in ihrer Gesamtheit nur auf der Basis verbindlicher und belastbarer Kooperationsstrukturen bewältigen können. Sie stehen in der Verantwortung, gemeinsam organisatorische, rechtliche und finanzielle Rahmenbedingungen $\mathrm{zu}$ etablieren, um eine verlässliche Informations- und Forschungsinfrastruktur mitzugestalten und durch Bündelung von Ressourcen die Entwicklungsdynamik und die nachhaltige Erbringung ihrer Dienste für die Wissenschaft abzusichern. ${ }^{1}$

Vor diesem Hintergrund ist es erforderlich, jenseits bereits etablierter Kooperationsmodelle auf regionaler Ebene oder bei der quelloffenen, kooperativen Entwicklung spezifischer Infrastrukturen und Werkzeuge neue Wege der Vernetzung auszuloten. Dabei kann die Kooperation von wissenschaftlichen Bibliotheken mit ähnlicher Zielgruppenstruktur gerade im Hinblick auf die konkreten Bedarfe bestimmter Fachdisziplinen ein vielversprechender Ansatz sein. Der im Jahre 2014 gestartete regelmäßige Austausch der Bibliotheken der TU9 nutzt die bereits erfolgreich etablierte Marke TU9, um durch die Kooperation der führenden deutschen Technik-Bibliotheken innovative und nachhaltige Dienstleistungen für die Community der Ingenieur- und Naturwissenschaften zu entwickeln.

\footnotetext{
1 Die zentrale Bedeutung nachhaltiger kooperativer Strukturen wird in zwei jüngst veröffentlichten Papieren adressiert: „Wissenschaftliche Bibliotheken 2025“, Strategiepapier der Sektion 4 im Deutschen Bibliotheksverband e. V. http://www.bibliotheksverband.de/fileadmin/user_upload/Sektionen/sektion4/Publikationen/WB2025_Endfassung_endg.pdf (10.4.2018) und „Förderung von Informationsinfrastrukturen für die Wissenschaft. Ein Positionspapier der Deutschen Forschungsgemeinschaft“. Bonn, 15. März 2018. http://www.dfg.de/download/pdf/foerderung/programme/lis/positionspapier_informationsinfrastrukturen.pdf (10.4.2018).
}

ว Open Access. (C) 2018 Katrin Stump, publiziert von De Gruyter. (c) Br-Nc-ND Dieses Werk ist lizenziert unter der Creative Commons Attribution-NonCommercial-NoDerivatives 4.0 Lizenz. 


\section{TU9 - Excellence in Engineering and the Natural Sciences - Made in Germany}

Bereits im Jahre 2003 riefen die Präsidenten der neun ältesten Technischen Universitäten in Aachen, Berlin, Braunschweig, Darmstadt, Dresden, Hannover, Karlsruhe, München und Stuttgart einen informellen Kreis ins Leben, bevor am 26. Januar 2006 eine feste Rechtsform gefunden und der Verein „TU9 German Institutes of Technology“ gegründet wurde. „Wir wollen uns damit als nationaler Ansprechpartner von Gesellschaft, Wirtschaft und Politik etablieren und uns noch stärker für die Belange der universitären Ingenieurausbildung stark machen“, erklärte der damalige Präsident des Karlsruher Instituts für Technologie, Prof. Horst Hippler, der zum TU9-Präsidenten gewählt wurde. ${ }^{2}$

Organisiert als Verein besteht TU9 aus der Mitgliederversammlung und dem Vorstand, dem die Präsidentin oder der Präsident sowie die Vizepräsidentin oder der Vizepräsident angehören, die beide für die Dauer von zwei Jahren mit der Möglichkeit der einmaligen Wiederwahl gewählt werden. Unterstützt werden Vorstand und Vereinsmitglieder von einer Geschäftsstelle, die in Berlin angesiedelt ist.

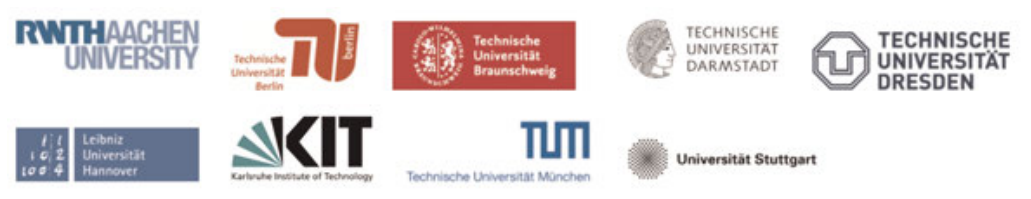

Abb. 1: Mitglieder der TU9. ${ }^{3}$

Übergeordnete Aufgabe des Zusammenschlusses ist die Förderung von Wissenschaft und Forschung in den Ingenieur- und Naturwissenschaften sowie die Förderung eines positiven Technikbewusstseins in der Gesellschaft. ${ }^{4}$ Markenzeichen der TU9 unter dem Motto „Excellence in Engineering and the Natural Sciences - Made in Germany“ sind neben der Forschungsstärke und ihrer internationalen Ausrichtung auch ihre Praxisnähe und die Interdisziplinarität in der Forschung und beim Studienangebot. Mit über 270000 Studierenden sind derzeit ca. $10 \%$ aller in Deutschland Studierenden an den TU9 eingeschrieben. Sie

2 TU9 institutionalisiert, Pressemitteilung der TU9 vom 26. Januar 2006. http://www.tu9.de/ presse/presse_752.php (28.1.2018).

3 http://www.tu9.de/728.php (28.1.2018).

4 Vgl. http://www.tu9.de/tu9/1473.php (28.1.2018). 
bringen zudem rund $50 \%$ der Universitätsabsolventinnen und -absolventen sowie Promotionen in den Ingenieurwissenschaften in Deutschland hervor.

Von Beginn an nutzte TU9 ihre Allianz, um aktuelle wissenschafts-, hochschul- und bildungspolitische Themen gemeinsam zu adressieren, sei es vor gut zehn Jahren zu den Bologna-Reformen ${ }^{5}$ und zum Erhalt des akademischen Grades „Diplom-Ingenieur“6 oder jüngst zu attraktiven Arbeitsbedingungen für den wissenschaftlichen Nachwuchs ${ }^{7}$ und zur Qualitätssicherung und Einhaltung wissenschaftlicher Standards bei Promotionen in Kooperation mit der Wirtschaft. ${ }^{8}$ Daneben vermarkten sich die TU9 selbstbewusst als „German Engineering“ im Ausland, vor allem durch die gemeinsame Präsenz auf internationalen Konferenzen ${ }^{9}$ und Messen, durch gezielte Studierendenwerbung ${ }^{10}$ oder durch Kooperationsvereinbarungen mit ähnlichen ausländischen Allianzen. ${ }^{11}$ Wie erfolgreich sich die TU9 als Sprachrohr der Technikwissenschaften positionieren konnten, zeigte nicht zuletzt die enorme Präsenz hochrangiger Persönlichkeiten aus Wissenschaft, Politik und Wirtschaft beim Symposium zur Zukunft des Wissenschafts- und Innovationsstandortes Deutschland anlässlich des zehnjährigen Bestehens der TU9. ${ }^{12}$

5 TU9-Präsident Prof. Dr. Horst Hippler fordert, Pressemitteilung der TU9 vom 20. Juli 2006. http://www.tu9.de/presse/presse_758.php (28.1.2018).

6 Bologna-Reform und „Dipl.-Ing.“ sind vereinbar: Österreich macht es vor! Pressemitteilung der TU9 vom 17. April 2010. http://www.tu9.de/media/docs/tu9/20100417_PM_TU9_Beschluss_Dipl_Ing.pdf (28.1.2018).

7 Leitlinien für attraktive Beschäftigungsverhältnisse an TU9-Universitäten. Berlin, 8. Mai 2015. http://www.tu9.de/media/docs/20150509_Leitlinien_fuer_attraktive_Beschaeftigungsverhaeltnisse_an_TU9.pdf (28.1.2018).

8 TU9-Eckpunktepapier zur Qualitätssicherung und Einhaltung wissenschaftlicher Standards bei Promotionen in Kooperation mit der Wirtschaft, Pressemitteilung der TU9 vom 13. Dezember 2017. http://www.tu9.de/presse/presse_7086.php (28.1.2018).

9 TU9 auf der 69. NAFSA Annual Conference \& Expo in L. A., California (USA), Pressemitteilung der TU9 vom 30. Mai 2017. http://www.tu9.de/presse/presse_7065.php (28.1.2018).

10 TU9 wirbt in Chile und Kolumbien um internationale MINT-Studierende, Pressemitteilung der TU9 vom 20. März 2017. http://www.tu9.de/presse/presse_7046.php (28.1.2018).

11 TU9 empfängt Nordic Five Tech - die Allianz der Technischen Universitäten Nordeuropas, Pressemitteilung der TU9 vom 23. November 2017. http://www.tu9.de/presse/presse_7083.php (28.1.2018).

12 „Sprachrohr der Technikwissenschaften“, Pressemitteilung der TU9 vom 12. Oktober 2016. http://www.tu9.de/presse/presse_6984.php (28.1.2018). 


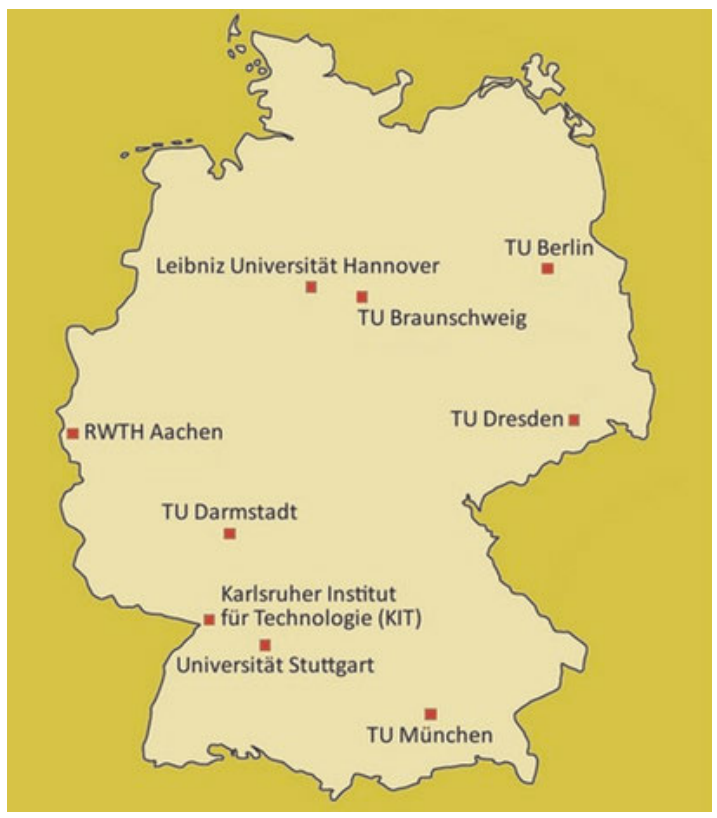

Abb. 2: TU9-Universitäten in Deutschland. ${ }^{13}$

Im Oktober 2014 erfuhr mit der Veröffentlichung des ersten TU9-MOOCs ein weiteres Projekt große Aufmerksamkeit, ${ }^{14}$ setzte sich die TU9 doch zum Ziel, ihr Lehrangebot in den Ingenieurwissenschaften mit innovativen Formaten kooperativ $\mathrm{zu}$ verbessern und $\mathrm{zu}$ erweitern und nicht zuletzt dadurch auch die Bekanntheit der Marke TU9 zu stärken. Unter dem Branding MOOC@TU9 werden mittlerweile fünf deutsch- bzw. englischsprachige Kurse zur Kommunikationsakustik, Verkehrsökologie, zum zeitgenössischen Städtebau, zu Flugtriebwerken und zum Entwicklungsprozess technischer Systeme angeboten, die von renommierten Wissenschaftlerinnen und Wissenschaftlern aus mindestens zwei TU9-Standorten entwickelt wurden. ${ }^{15} \mathrm{Ca} .3000$ Personen nahmen bereits an den

13 http://www.tu9.de/728.php (wie Anm. 3; 28.1.2018).

14 TU9 MOOC zu „German Engineering“: Startschuss am 20.10.2014, Pressemitteilung der TU9 vom 15. Oktober 2014. http://www.tu9.de/presse/presse_6674.php (28.1.2018). Der Pilotkurs „Discover Excellence in Engineering and the Natural Science - Made in Germany“ fand als gemeinsame, englischsprachige Ringvorlesung aller TU9 über insgesamt neun Wochen statt. 15 MOOC@TU9: Gebündeltes ingenieurwissenschaftliches Know-how für jedermann, Pressemitteilung der TU9 vom 17. Oktober 2016. http://www.tu9.de/presse/presse_7004.php. Zu den einzelnen MOOCs siehe http://www.tu9.de/projekte/6980.php (28.1.2018). Gefördert wird das Projekt durch Mittel der Programminitiative MINTernational der Daimler und Benz Stiftung, des Daimler Fonds und des Stifterverbands. 
zum Teil curricular verankerten Kursen teil, ${ }^{16}$ wobei auch Studierende außerhalb der TU9 Credits erwerben können.

\section{Der Anfang ist gemacht! Die Bibliotheken der TU9 vereinbaren eine engere Zusammenarbeit}

Unter dem Dach der im deutschen Hochschulwesen bestens bekannten Marke TU9 vernetzten sich im Laufe der Zeit verschiedene Personenkreise der neun Universitäten wie die Chief Information Officer oder die EU-Forschungsreferate, sodass die Sächsische Landesbibliothek - Staats- und Universitätsbibliothek Dresden (SLUB) eine Zusammenarbeit auch auf bibliothekarischer Ebene anregte. Im Sommer 2014 loteten die Direktorinnen und Direktoren der TU9-Bibliotheken $^{17}$ aussichtsreiche Themenfelder und die Anschlussfähigkeit laufender Infrastrukturprojekte der einzelnen Häuser für ihre Kooperation aus. Zudem galt es, grundlegende Ziele und Organisation der Zusammenarbeit festzulegen und die Interaktion mit den jeweiligen Hochschulleitungen sowie dem TU9-Präsidium und der TU9-Geschäftsstelle zu klären. ${ }^{18}$

Mit den Themenfeldern Forschungsdatenmanagement, Lizenzierung und Open Access wurden Schwerpunkte für gemeinsame Aktivitäten identifiziert. Darüber hinaus verabredete man, nicht ausschließlich bei einem Informationsund Erfahrungsaustausch $\mathrm{zu}$ verharren, sondern Strategien und infrastrukturelle Lösungen der einzelnen Häuser vorzustellen, um so Best Practice zu fördern, Synergien zu nutzen und bestenfalls kooperativ Infrastrukturen und Werkzeuge zu entwickeln. Erstes sichtbares Zeichen der neuen Kooperation war die gemeinsame Präsenz der TU9-Bibliotheken auf der IATUL-Tagung ${ }^{19}$ im Juli 2015 in Hannover mit einem eigenen Stand. Bibliotheksbeschäftigte präsentier-

16 Vgl. http://www.tu9.de/projekte/6515.php (28.1.2018).

17 Dazu gehören die Universitätsbibliotheken der RWTH Aachen, der TU Berlin und der TU Braunschweig, die Universitäts- und Landesbibliothek Darmstadt, die Sächsische Landesbibliothek - Staats- und Universitätsbibliothek Dresden, die TIB - Leibniz-Informationszentrum Technik und Naturwissenschaften und Universitätsbibliothek in Hannover, die KIT-Bibliothek Karlsruhe sowie die Universitätsbibliotheken der TU München und der Universität Stuttgart. 182016 wurde vereinbart, dass der Sprecher der Bibliotheken turnusmäßig mit dem TU9-Präsidentenamt wechselt. Daher übernahm zunächst der Direktor der Universitäts- und Landesbibliothek Darmstadt diese Funktion, die zu Beginn des Jahres 2018 mit dem Wechsel des Präsidentenamtes an den Rektor der Universität Stuttgart auf die dortige Universitätsbibliothek überging.

19 The International Association of University Libraries: https://www.iatul.org/. 
ten Projekte aus ihren Einrichtungen und demonstrierten so die Leistungsfähigkeit der TU9-Bibliotheken.

\section{Gemeinsame Aktivitäten im Bereich Forschungs- datenmanagement}

Das Management von Forschungsprimärdaten sowie deren Archivierung und Nachnutzung spielen gerade in den stark datengetriebenen Ingenieur- und Naturwissenschaften eine herausragende Rolle. Die daraus resultierenden großen Herausforderungen im Hinblick auf den Aufbau von Forschungsdateninfrastrukturen sowie Beratungs- und Unterstützungsservices lassen sich nur durch das verbindliche und belastbare Zusammenspiel von Datenproduzenten, Bibliotheken und Rechenzentren bewältigen.

Für die nachhaltige Entwicklung und Etablierung von Services für die technisch-naturwissenschaftlichen Disziplinen erweist sich die Kooperation der TU9-Bibliotheken als besonders vielversprechend. Das erste Treffen der zuständigen Expertinnen und Experten zu Jahresbeginn 2017 identifizierte als vordringliches Thema die Erstellung bzw. die Weiterentwicklung von Weiterbildungskonzepten zur Professionalisierung im Umgang mit Forschungsdaten im Bereich der Nachwuchsförderung. Der eigene Kompetenzausbau aller thematisch Verantwortlichen in Form einer Train-the-Trainer-Weiterbildung bildete den Auftakt für die weitere konzeptionelle Arbeit, die in der Bereitstellung nachnutzbarer Kursformate und -materialien mündete.

Stehen derzeit vor allem die Nachwuchsforschenden vor und nach der Promotion im Mittelpunkt der Weiterbildungsaktivitäten, so wird künftig die curricular verankerte Entwicklung von Data-Science-Kompetenzen in Bachelor- und Masterstudiengängen eine zentrale Rolle spielen. So fordert der Rat für Informationsinfrastrukturen in seinen 2016 erschienenen Empfehlungen, „Module zur Vermittlung von Informationskompetenz und Datenmanagementkenntnissen in das gesamte Spektrum bestehender Studiengänge zu integrieren““. ${ }^{20}$ Im gleichen Sinne formuliert der Stifterverband im jüngst erschienenen Hochschul-BildungsReport 2020 als eines von acht Zielen universitärer Bildungspolitik die Sicherung von Datenanalysekompetenzen in allen Disziplinen und den Ausbau von Data Science an den Hochschulen. Konkret empfiehlt er die „Einrichtung

20 Rat für Informationsinfrastrukturen: Leistung aus Vielfalt. Empfehlungen zu Strukturen, Prozessen und Finanzierung des Forschungsdatenmanagements in Deutschland. Göttingen 2016, S. 50. http://www.rfii.de/?wpdmdl=1998 (28.1.2018). 
von Data-Science-Education-Programmen für die Bachelorstudiengänge an Hochschulen, die grundlegende Datenanalysefähigkeiten für alle Fächer vermitteln und an denen alle Studierenden teilnehmen sollten“ sowie „[g]ezielte Kooperationen von Hochschulen und Unternehmen bei der Vermittlung von Datenanalysekompetenzen“. ${ }^{21}$

Für die Bewältigung dieser Herausforderungen in den technisch-naturwissenschaftlichen Disziplinen kann die sich entwickelnde Kooperation der Forschungsdatenverantwortlichen der TU9-Bibliotheken gute Anknüpfungspunkte bieten. Um auf der Basis geeigneter didaktischer Konzepte den Kompetenzerwerb bei den Studierenden abzusichern, müssen diese Aktivitäten zukünftig mit denen der Informationskompetenzvermittlung verschränkt werden, zumal hier langjährige Erfahrung mit Lehrformaten wie E-Learning, Blended Learning oder Gamification und deren Einsatz in Großgruppen vorliegt.

\section{Gemeinsame Aktivitäten in den Bereichen Lizenzierung und Open Access}

Um den spezifischen Bedarfen der TU9 an Informationsmedien gerecht zu werden, wurden frühzeitig der Austausch und die Abstimmung der TU9-Bibliotheken im Bereich Lizenzierung initiiert. Seit 2016 treffen sich die Lizenzierungsverantwortlichen einmal jährlich und beraten sich zu konkreten Produkten, Lizenzierungspraktiken, neuen Publikationsformaten und inneruniversitären Etatverteilungsmodellen, aber auch zu aktuellen Themen wie dem Projekt DEAL $^{22}$ und zu Open-Access-Transformationsverträgen.

Mit der TIB - Leibniz-Informationszentrum Technik und Naturwissenschaften und Universitätsbibliothek ist in diesem Kreis auch die Deutsche Zentrale Fachbibliothek für Technik, Architektur, Chemie, Informatik, Mathematik und Physik vertreten, die zahlreiche nationale Konsortien verhandelt. Ausgehend vom konkreten Bedarf mehrerer TU9-Bibliotheken konnte z. B. für das Produkt DETAIL inspiration ein nationales Konsortium formiert werden, dem sich zahlreiche weitere Bibliotheken anschlossen und das nach einjähriger Laufzeit für

21 Stifterverband für die Deutsche Wissenschaft: Hochschul-Bildungs-Report 2020. Höhere Chancen durch höhere Bildung? Jahresbericht 2017/18 - Halbzeitbilanz 2010 bis 2015. Essen 2017, S. 3. https://www.stifterverband.org/download/file/fid/5047 (28.1.2018).

22 Projekt DEAL - Bundesweite Lizenzierung von Angeboten großer Wissenschaftsverlage: https://www.projekt-deal.de/aktuelles/ (10.4.2018). 
den Zeitraum 2018 bis 2020 in eine DFG-geförderte Allianzlizenz überführt werden konnte.

Eine gemeinsame Strategie der TU9-Bibliotheken im Bereich Lizenzierung wird derzeit aufgrund diverser Bindungen an regionale Konsortien als nicht zielführend erachtet. Jedoch zeigt das Beispiel der eben erwähnten Datenbank, dass die TU9-Biblioheken aufgrund der fachlichen Ähnlichkeit ihrer Stakeholder Keimzellen für die Konsortialbildung von Produkten sein können, die bislang nur lokal oder noch gar nicht lizenziert sind, und auf diesem Wege die Informationsversorgung für die Ingenieur- und Naturwissenschaften optimiert wird.

Ein weiteres, sehr dynamisches Kooperationsfeld etablierte sich zu Open Access. Das Ziel, den Output der TU9 an Open-Access-Publikationen auf der TU9-Webseite schaufensterartig darzustellen, wurde durch Kooperation mit der Bielefelder Suchmaschine BASE verwirklicht. ${ }^{23}$ Auf deren Basis wird eine Abfrage der Bibliotheksrepositorien generiert, Suchschlitz und Trefferausgabe wurden in den Webauftritt der TU9 integriert. ${ }^{24}$ Im nächsten Schritt wird man ausloten, inwieweit sich die vorhandene Datenbasis eignet, um mit innovativen Methoden die Publikationsdaten nutzbringend zu erschließen und vernetzen.

Etabliert hat sich mittlerweile auch der regelmäßige Austausch der OpenAccess-Beauftragten der TU9-Bibliotheken, der Themen wie die Bewirtschaftung von Open-Access-Publikationsfonds und deren Verstetigung sowie bibliometrische Dienstleistungen adressiert. Unter der Marke TU9 kommuniziert man aber auch gemeinsam Defizite beim Handling institutioneller Open-Access-Mitgliedschaften an die Verlagsverantwortlichen und fordert den Abbau dieser Missstände.

Aus den guten Arbeitsbeziehungen der Bibliotheken erwuchs zuletzt die erfolgreiche Beantragung des Projekts „OpenIng - Open Access und Open Educational Resources in den Ingenieurwissenschaften“ im Rahmen der BMBF-Ausschreibung „Förderrichtlinie des freien Informationsflusses in der Wissenschaft - Open Access“. Da Open Access in den Ingenieurwissenschaften vergleichsweise noch wenig verbreitet ist, zielt OpenIng auf der Basis der zu erforschenden Ursachen auf die Entwicklung und Evaluierung neuer nachnutzbarer Services und Unterstützungsangebote. ${ }^{25}$ Das Projekt ist in die Zusammen-

23 Bielefeld Academic Search Engine: https://www.base-search.net (10.4.2018).

24 Open-Access-Publikationen aller TU9-Universitäten mit einem Klick, Pressemitteilung der TU9 vom 1. Februar 2018. https://www.tu9.de/presse/presse_7103.php. Der Suchschlitz findet sich auf der TU9-Webseite im Bereich Forschung: https://www.tu9.de/forschung/ (1.2.2018).

25 Open Access in den Ingenieurwissenschaften: BMBF fördert Projekt OpenIng, Pressemitteilung der Technischen Universität Darmstadt vom 25. Januar 2018. https://idw-online.de/de/ news688108 (28.1.2018). 
arbeit der Bibliotheken der führenden deutschen Technischen Universitäten eingebettet und wird unter Federführung der TU Darmstadt realisiert. ${ }^{26}$ Wie an diesem Projekt ersichtlich ist es nicht zwingend erforderlich, dass immer alle neun Bibliotheken gemeinsam auftreten, sondern sich auch Einrichtungen bioder trilateral zu konkreten Kooperationsprojekten zusammenschließen können. Da sie mit den anderen Bibliotheken gegebenenfalls Letters of Intent vereinbaren, in jedem Fall aber im Kreise der Leitungsebene regelmäßig über die Projekte berichten, ist die Anschlussfähigkeit für die nicht unmittelbar am Projekt beteiligten Einrichtungen gewährleistet.

\section{BibHop - Das Austauschprogramm der TU9- Bibliotheken}

Von Beginn an war seitens der Bibliotheksleitungen eine umfassende Vernetzung der Einrichtungen intendiert, die explizit nicht nur auf die Direktionsebene zielt, sondern - wie bereits an einigen Beispielen erläutert - Austauschformate verschiedener Fachexpertisen fördert. Um Möglichkeiten der weiteren Vernetzung zu eröffnen, den kollegialen Erfahrungs- und Informationsaustausch $\mathrm{zu}$ intensivieren, sich gegenseitig $\mathrm{zu}$ inspirieren und Impulse für die Selbstreflektion und zur Überwindung der eigenen „Betriebsblindheit“ zu setzen, wurde auf Initiative der SLUB ein niedrigschwelliges Hospitationsprogramm zwischen den TU9-Bibliotheken aufgelegt, das sich an die Bibliotheksbeschäftigten aller Ebenen richtet. ${ }^{27}$ Weiteres Ziel ist die Stärkung eines Zusammengehörigkeitsgefühles der TU9-Bibliotheken, das auf der Basis intensivierter Arbeitsbeziehungen die Entwicklung und Durchführung gemeinsamer Projekte beflügelt.

Für die Programmkonzeption und deren organisatorische Realisierung zeichneten die Fortbildungsbeauftragten bzw. Personalentwicklerinnen oder -entwickler der neun Bibliotheken verantwortlich. Mit je acht Plätzen in den neun Bibliotheken wird pro Jahr insgesamt 72 Personen die Möglichkeit geboten, eine TU9-Bibliothek eigener Wahl im Umfang von zwei Tagen inklusive Anund Abreise kennenzulernen. Jede Bibliothek listet in einem Themenkatalog die

26 Die Gesamtkoordination liegt bei der Universitäts- und Landesbibliothek Darmstadt und der Hochschuldidaktischen Arbeitsstelle der TU Darmstadt. Weiterhin sind die Universitätsbibliotheken Braunschweig und Stuttgart beteiligt, verschiedene TU9 steuerten zudem Letters of Intent bei.

27 Als Vorbild diente das Goportis-Hospitationsprogramm der drei Deutschen Zentralen Fachbibliotheken. 
spezifischen Angebote des eigenen Hauses auf, sodass sich die Gäste ein ihrem Arbeitsgebiet und ihren Interessen entsprechendes, individuelles Programm zusammenstellen können. Die Bibliotheksführung und das Abendessen am ersten Tag bilden die gemeinsame Klammer der Hospitationen. Nach zwei Jahren wird das Austauschprogramm evaluiert und gemeinsam über seine Fortsetzung entschieden. Nach dem ersten Durchgang im Jahr 2017 gaben die beteiligten Beschäftigten sowohl auf Gäste- als auch auf Gastgeberseite positives Feedback, da sie den kollegialen Austausch gerade auch für die Personenkreise, die nicht so häufig an externen Fortbildungen teilnehmen, als sehr förderlich einschätzten.

\section{Gemeinsame Lobbyarbeit unter der Marke TU9}

Nachdem die TU9 sich regelmäßig zu aktuellen hochschulpolitischen Themen positionieren, nutzten ihre Bibliotheken diese Allianz für die Adressierung von zwei Themen, die 2017 im Fokus sowohl von Universitäts- und Bibliotheksleitungen als auch von Forschenden, Studierenden und der Wissenschaftspolitik standen.

Spätestens seit Mitte 2016 erfuhr das Projekt DEAL zunächst vor allem national, seit Anfang 2017 zunehmend auch international enormen Widerhall. ${ }^{28}$ Unter den ca. 65 deutschen Einrichtungen, die ihre Elsevier-Verträge bereits 2016 gekündigt hatten und sich mit Beginn des Jahres 2017 in vertragslosem Zustand befanden, waren auch vier der TU9. Die zu diesem Zeitpunkt bereits etablierte Kooperation der Bibliotheken, die sich ebenso in guten Beziehungen zum TU9-Präsidenten und zur Geschäftsstelle manifestierte, bildete eine ideale Ausgangsbasis für die Formulierung einer gemeinsamen Presseerklärung der TU9, die sich geschlossen für die Unterstützung des Projekts aussprach und grundsätzlich bilaterale Gespräche mit dem Verlag Elsevier ausschloss. ${ }^{29}$

Auch in den heftig geführten Auseinandersetzungen um die Verabschiedung des Urheberrechts-Wissensgesellschafts-Gesetzes bezog die TU9 eindeutig Position, indem sie sich geschlossen hinter die Argumentation der Hochschul-

28 Vgl. https://www.projekt-deal.de/pressespiegel/ (28.1.2018).

29 TU9 spricht sich geschlossen für die bundesweite Lizenzierung von Angeboten großer Wissenschaftsverlage aus, Pressemitteilung der TU9 vom 4. April 2017. http://www.tu9.de/presse/ presse_7047.php (28.1.2018). 
rektorenkonferenz ${ }^{30}$ stellte und versuchte, ihren Einfluss im Sinne eines zeitgemäßen und praktikablen Urheberrechts geltend zu machen.

\section{Ausblick}

Die Kooperation der TU9-Bibliotheken hat sich in den letzten drei Jahren vielversprechend entwickelt und zeigt auf ausgewählten Themenfeldern bereits erste sichtbare Erfolge. Auch wurden mit der digitalen Langzeitarchivierung und dem Thema Personalrecruiting schon weitere Felder identifiziert, die sich für eine intensivere Betrachtung möglicher gemeinsamer Aktivitäten anbieten.

Es kann allerdings nicht verschwiegen werden, dass die TU9-Bibliotheken durch ihren zum Teil über die eigene Universität hinausreichenden Auftrag und ihre Einbindung in regionale, nationale oder internationale Aktivitäten ebenso eigene, von den TU9 unabhängige Agenden verfolgen und gelegentlich auch in Konkurrenz zueinander stehen.

Um jedoch das spezifische Bedarfsspektrum der technisch-naturwissenschaftlichen Fachdisziplinen zu adressieren und für diese Fachcommunitys den Ausbau einer verlässlichen Informations- und Forschungsinfrastruktur voranzutreiben, sind verbindliche Kooperationen leistungsstarker Einrichtungen unerlässlich. In diesem Sinne sollten die TU9-Bibliotheken durch die zielstrebige Intensivierung ihrer Zusammenarbeit einen wichtigen Knoten in dem sich formierenden Netzwerk überregional agierender Infrastrukturen bilden und für ihre Zielgruppen kooperativ attraktive Dienste und Werkzeuge entwickeln, die sie in Forschung, Lehre und Studium optimal unterstützen.

Der Anfang ist gemacht, nun gilt es, die Mühen der Ebene zu meistern!

30 HRK-Präsident zur Urheberrechtsreform: Kompromisslos für Digitalisierung, Pressemitteilung der HRK vom 23. Juni 2017. https://www.hrk.de/presse/pressemitteilungen/pressemitteilung/meldung/hrk-praesident-zur-urheberrechtsreform-kompromisslos-fuer-digitalisierung4178/ (28.1.2018). 